\title{
Review Article \\ External Application of Traditional Chinese Medicine for Venous Ulcers: A Systematic Review and Meta-Analysis
}

\author{
Xin Li, ${ }^{1}$ Qing-qing Xiao, ${ }^{1}$ Kan Ze, ${ }^{1}$ Su Li, ${ }^{1}$ Yi-fei Wang, ${ }^{1}$ Min Zhou, ${ }^{1}$ Qin-tong Yang, ${ }^{2}$ \\ Fu-lun Li, ${ }^{1}$ and Bin $\mathrm{Li}^{1}$ \\ ${ }^{1}$ Department of Dermatology, Yueyang Hospital of Integrated Traditional Chinese and Western Medicine, \\ Shanghai University of Traditional Chinese Medicine, Shanghai 200437, China \\ ${ }^{2}$ Department of Vessel, Shanghai TCM-Integrated Hospital, Shanghai University of Traditional Chinese Medicine, \\ Shanghai 200082, China \\ Correspondence should be addressed to Fu-lun Li; drlifulun@163.com and Bin Li; 18930568129@163.com
}

Received 22 May 2015; Revised 14 August 2015; Accepted 23 August 2015

Academic Editor: Yibin Feng

Copyright (C) 2015 Xin Li et al. This is an open access article distributed under the Creative Commons Attribution License, which permits unrestricted use, distribution, and reproduction in any medium, provided the original work is properly cited.

Objective. To evaluate the effectiveness of external application of traditional Chinese medicine (EA-TCM) on venous ulcers. Methods. Seven databases were searched until April 2015 for randomized controlled trials (RCTs) of EA-TCM for venous ulcers. Risk of bias was assessed using Cochrane Handbook guidelines. Study outcomes were presented as risk ratios (RRs) for dichotomous data or mean differences (MDs) for continuous data. Results. Sixteen of 193 potentially relevant trials met the inclusion criteria; however, their methodological qualities were low. Comparison of the same intervention strategies revealed significant differences in total effectiveness rates between EA-TCM and conventional therapy groups ( $\mathrm{RR}=1.22,95 \%$ confidence interval $[\mathrm{CI}]=1.16-$ 1.29 , and $P<0.00001)$. Compared to conventional therapy, EA-TCM combined with conventional therapy had a superior total effectiveness rate $(\mathrm{RR}=1.11,95 \% \mathrm{CI}=1.04-1.19$, and $P=0.003)$. There were no significant differences in recurrence rates during followup and final pain measurements between the experimental and those in the control groups $(\mathrm{RR}=0.86,95 \% \mathrm{CI}=0.31-2.39$, and $P=0.85$; $\mathrm{MD}-0.75,95 \% \mathrm{CI}=-2.15-0.65$, and $P=0.29$ ). Conclusion. The evidence that EA-TCM is an effective treatment for venous ulcers is encouraging, but not conclusive due to the low methodological quality of the RCTs. Therefore, more high-quality RCTs with larger sample sizes are required.

\section{Introduction}

The most commonly diagnosed ulcer of the lower extremities, venous leg ulcerations, occurs in approximately 500,000 to 2 million people annually in the United States [1], with a prevalence as high as $4 \%$ in populations older than 65 years [2]. The treatment of venous ulcer disease requires significant resources and costs: in the United States, the overall cost is approximately 3 billion dollars per year [3]. The two main objectives of venous ulcer treatment are to heal the ulcer and to avoid ulcer recurrence [4]. Compression and debridement are the standard first-line clinical treatments. Secondline treatments, which involve a range of interventions, are considered when first-line treatments fail. However, until recently, there have not been widely accepted second-line treatment standards.
In addition to surgical treatments for venous ulcers, including more invasive open surgical procedures (i.e., venous ligation and stripping [5]), less invasive open surgical procedures (i.e., ambulatory conservative hemodynamic correction of venous insufficiency (CHIVA) [6] and ablative superficial venous surgery [7]), and less invasive endovenous surgical procedures (i.e., radiofrequency ablation [8] and endovenous laser [5]), therapies collectively known as traditional Chinese medicine (TCM) show a gradual and typically curative effect. In China, TCM has been used to treat human diseases for more than 2000 years. In the history of TCM, physicians have accumulated a tremendous amount of knowledge and experience in treating venous ulcers. As an integral part of TCM, external application of traditional Chinese medicine (EA-TCM) has been perceived as less expensive, safer, and more effective [9-11] than conventional 
therapies. There are numerous clinical trials regarding the use of EA-TCM for treatment of venous ulcers, with positive results; however, to our knowledge, the potential benefits of EA-TCM for patients with venous ulcers, to justify either their recommendation or their clinical role, have not been evaluated. In addition, a large number of studies could potentially be missed if literature searches are restricted to English-only sources [12]. Therefore, we conducted a systematic review to assess the effect of EA-TCM on venous ulcers.

\section{Materials and Methods}

2.1. Data Sources and Searches. To identify relevant randomized clinical trials (RCTs), two reviewers (X. Li and Q. Xiao) systematically searched the Medical Literature Analysis and Retrieval System Online (MEDLINE), Excerpta Medica data BASE (EMBASE), Cochrane Central Register, China National Knowledge Infrastructure database, Chinese Scientific Journals Full Text Database, Wanfang Data Knowledge Service Platform, and the Chinese Biomedical Literature Service System, using the search terms "venous ulcers," "venous leg ulcer," “TCM," "traditional Chinese herb," "herbal medicine," "ointment," and "randomized controlled trial." In this study, we included papers dating from the earliest citation in the databases until April 2015. The references of all selected publications and reviews were manually searched for further relevant articles. We did not limit publication languages and types, including conference proceedings, abstract-only articles, and theses, as long as they met our inclusion criteria.

\subsection{Study Selection}

2.2.1. Studies. RCTs were included. Quasi-RCTs, non-RCTs, or randomized trials with false randomization methods were excluded.

2.2.2. Participants. Patients diagnosed with venous ulcers based on any set of explicit criteria were included; other ulcers, such as pressure ulcers, were excluded. There were no set limitations on participant age, gender, or nationality.

2.2.3. Interventions. The focused experimental groups received either EA-TCM or EA-TCM combined with conventional therapy. We did not set limitations on dosages, formulations, routes of administration of the traditional Chinese herbs, or types of conventional therapy used.

Our comparison of TCM and conventional therapy included surgical treatment, endovenous surgical procedures, compression therapy, and topical and pharmacological treatment.

2.2.4. Control Group Treatments. Control groups were defined as patients who received any type of conventional therapy for venous ulcers, without TCM treatments.

2.2.5. Outcome Measures. The primary outcomes considered in this study were the total effectiveness rates for the duration of treatment, defined as the rate of change in ulcer size, absolute change in wound size, and number of wounds completely healed. We also evaluated recurrence rates, defined as the detection of new venous ulcers by clinical evaluation after followup. The secondary outcomes included quality of life, pain, and any adverse effects from the interventions.

Trials were excluded if any of the following factors were identified: (1) insufficient information concerning evaluation rates; (2) lack of EA-TCM treatment; (3) mixed interventions in the experimental group (e.g., EA-TCM combined with internal TCM); (4) animal trials.

2.3. Data Extraction. Two reviewers (K. Ze and S. Li) extracted data independently using a predefined data extraction form. Disagreements were resolved by discussion or consensus with a third reviewer (B. Li). The data extracted included the first author; study characteristics (i.e., year, duration, setting, and design); participant characteristics (i.e., mean age, sample size, and systemic therapy); external application of the experimental and control group treatments; measured outcomes. For studies with insufficient information, the reviewers contacted the primary authors, when possible, to acquire and verify the data.

2.4. Risk of Bias Assessment. The risk of bias in each study was assessed by two independent authors (X. Li and M. Zhou) using the Cochrane Risk of Bias tool [13]; disagreements were resolved either by consensus or by a third reviewer (B. Li).

2.5. Data Synthesis and Analyses. For meta-analysis, the total effectiveness rates of dichotomous data were pooled using risk ratios (RRs). All statistical analyses were performed using Review Manager 5.2.1 software (Cochrane Community, London, United Kingdom).

We compared the final results to assess the differences between experimental and control groups. Cochrane's $\chi^{2}$ and $I^{2}$ tests were used to assess the degree of heterogeneity between studies. There was considerable heterogeneity for $P$ values less than 0.10 , or $I^{2}$ value above $50 \%$, in the $\chi^{2}$ and $I^{2}$ tests, respectively [13]. In this case, a random-effects model was used in order to compute the global RR and MD. Otherwise, with $P$ values greater than 0.10 or $I^{2}$ less than $50 \%$, the between-study heterogeneity was not substantial, and the fixed-effect models were suitable. Clinical heterogeneity was assessed by reviewing the differences in the distribution of participants' characteristics among trials (i.e., age, gender, and duration of disorder and associated diseases).

\section{Results}

3.1. Study Selection. From a total of 193 titles, the full text of 75 potentially relevant studies was reviewed to confirm their eligibility. Among these 75 studies, 59 were excluded, including one non-RCT study, 23 with treatments that mixed interventions, nine with duplicate publication of data, 24 that compared treatment intervention with TCM, and two with no prescribed duration of treatment. Finally, 16 trials met the inclusion criteria (Figure 1). 


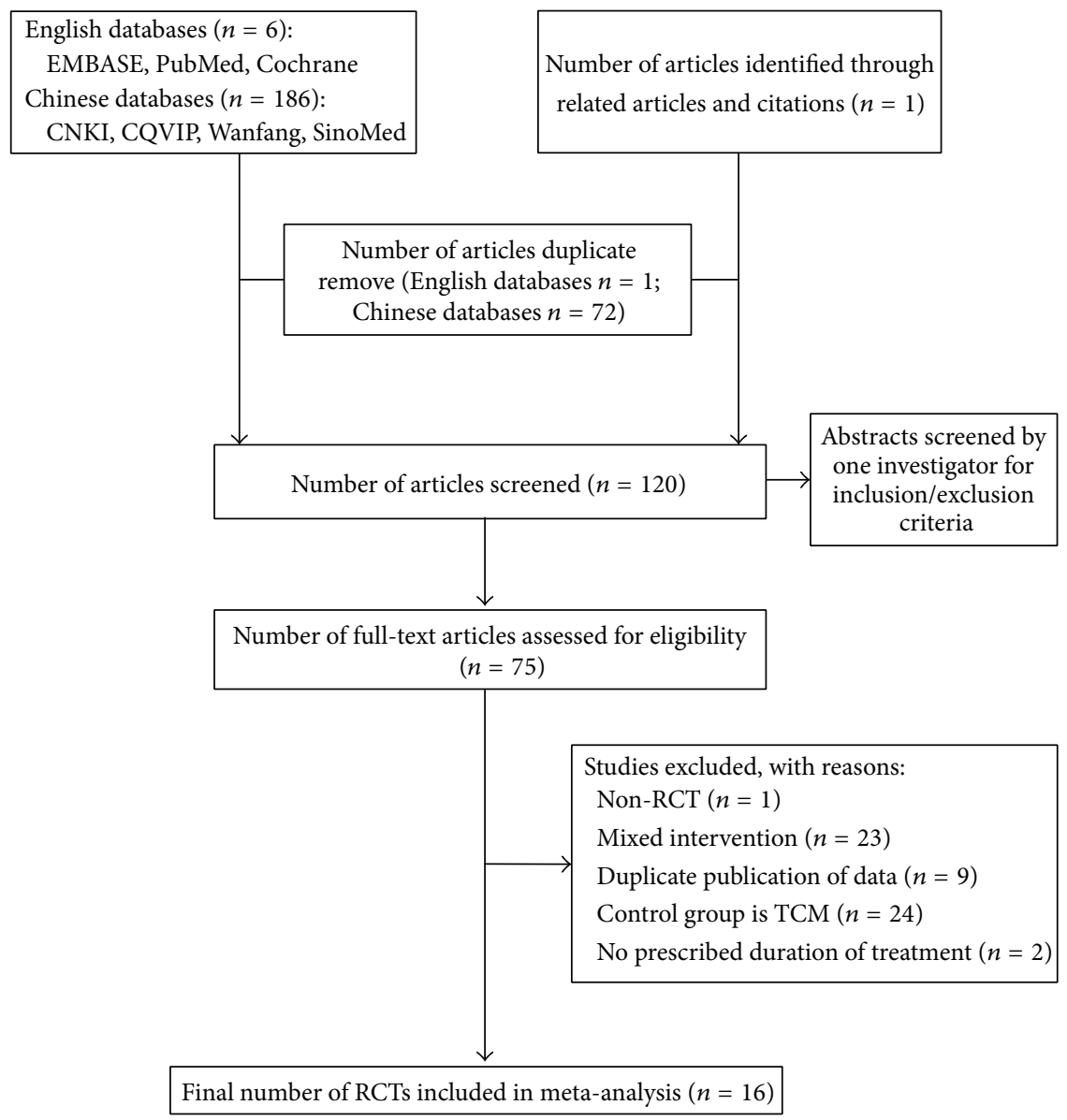

FIgURE 1: Summary of the literature identification and selection process. CNKI indicates the Chinese National Knowledge Infrastructure database; CQVIP, the Chinese Scientific Journals Full Text database; SinoMed, the Chinese Biomedical Literature Service System; TCM, traditional Chinese medicine; RCT, randomized clinical trials.

3.2. Study Characteristics. All of the 16 trials included in this study were published in Chinese. A total of 1269 participants were included in these trials, with 660 and 609 in the experimental and control groups, respectively. The sample sizes of these trials ranged from 51 to 164. Six trials reported on the adverse events in the experimental group [11, 14-18], while three performed patient followup [11, 14, 19] (Table 1).

The components and suppliers of the traditional Chinese herbs used in each trial varied. The most common form of EA-TCM, used in nine trials, was ointment, including SheXiangZhenZhu [20], KuiYangPing [9], ShengJi [19], moist exposed burn [15], ShengJiYuHong [11, 21], HongYou [18, 22], and FuFangSanHuang ointments [23]. Other forms of EATCM used in clinical trials were powders in three trials $[10,14$, 16], Chinese-herb external washing in three trials [17, 22, 24], paste in one trial [9], and oil in one trial [25] (Table 2).

3.3. Risk of Bias Assessment. The methodological quality of all included trials was poor (Figure 2). Although all these trials reported randomization, only three adequately described the randomization method: two with a random number table $[14,25]$ and one using clinic record numbers [9]. Moreover, none of the studies reported information such as allocation concealment or blinding of participants and study personnel; only one reported the details of the blinding of outcome assessment [16]. All of the relevant trials adequately addressed incomplete outcome data and selective reporting. We found no other biases in these trials; however, considering their poor methodological quality, we determined that an unclear risk of bias should be given to all the included trials.

\subsection{Primary Outcomes}

3.4.1. Total Effectiveness Rates of EA-TCM versus Conventional Therapy Based on the Same Intervention Strategies. The 11 RCTs contained 761 patients; the experimental and control groups received EA-TCM and conventional therapy, respectively. All subjects from the two groups received basic intervention strategies, including compression and debridement as first-line clinical treatment and surgical interventions (venous ligation and stripping $[15,26]$ and endovenous laser [18]) as second-line treatment. Pooling of the results from these trials showed a significant difference in the total effectiveness rate between the EA-TCM and conventional therapy groups $(\mathrm{RR}=1.22,95 \%$ confidence interval $[\mathrm{CI}]=$ 1.16-1.29, and $P<0.00001)$ using the fixed-effects model. 


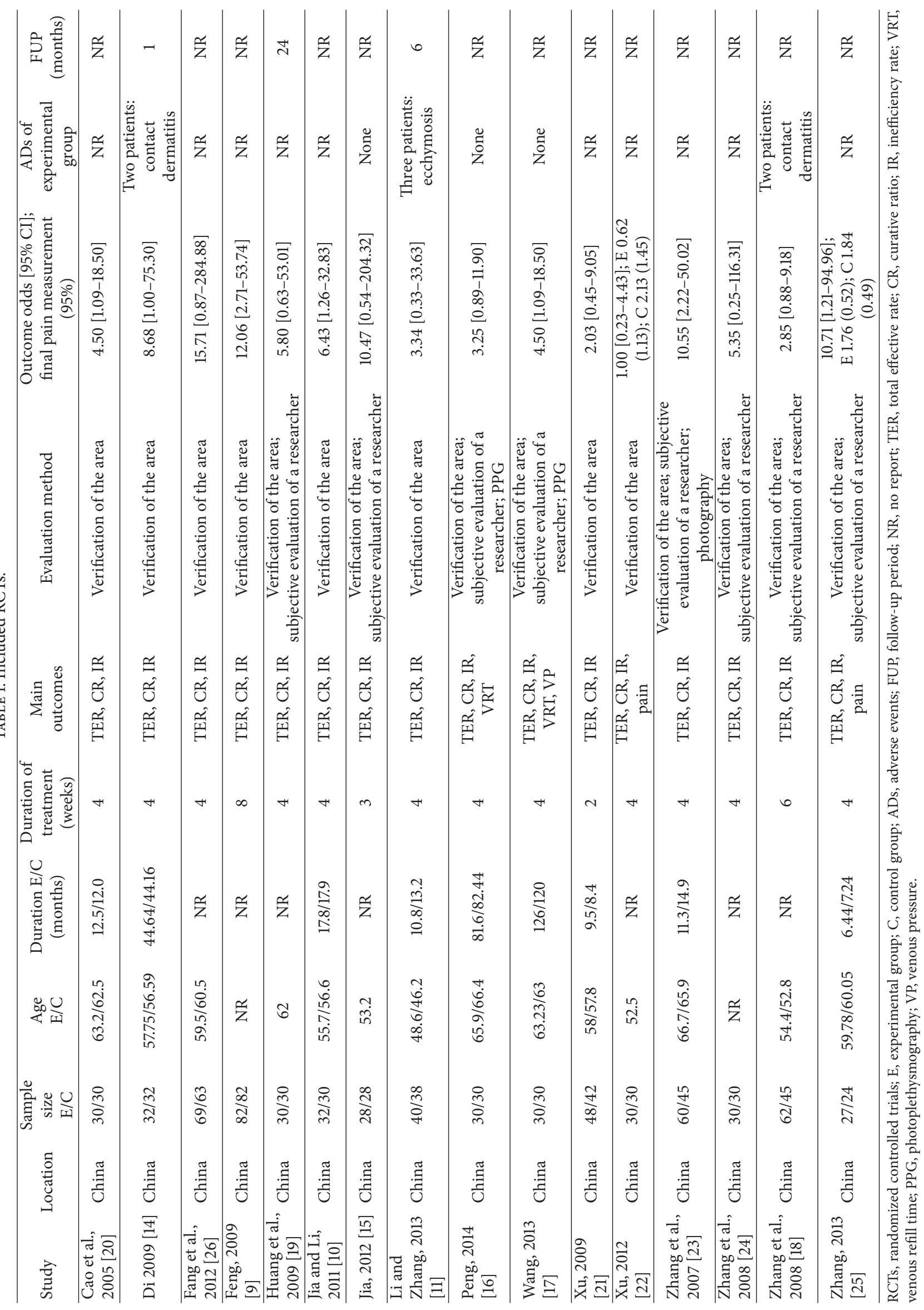



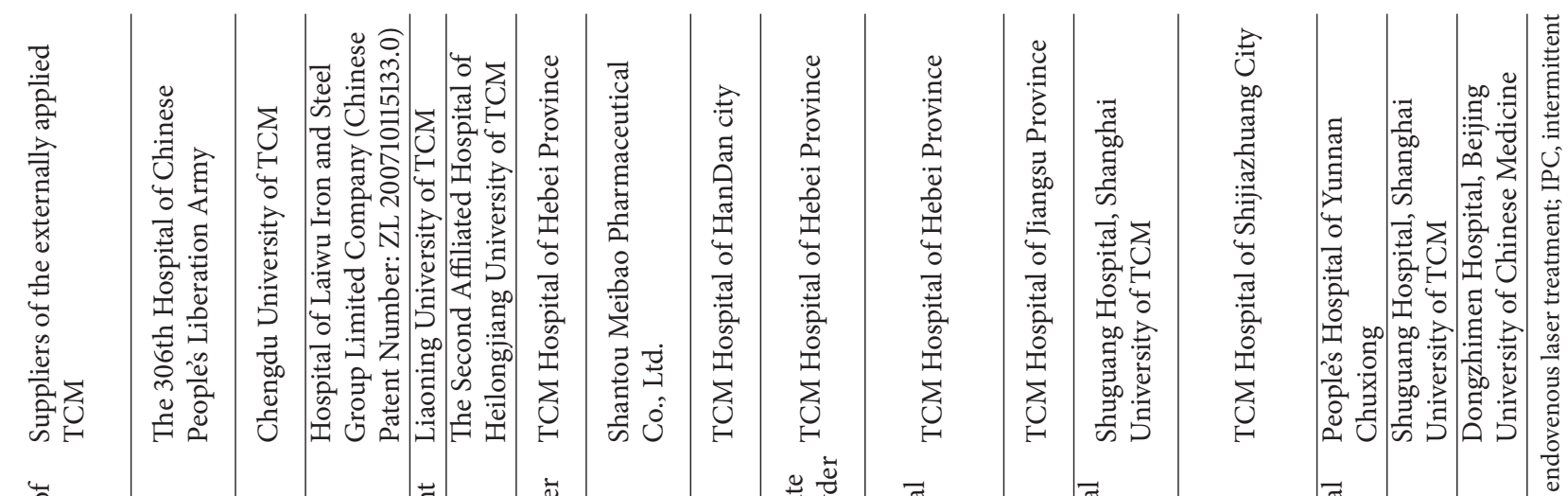

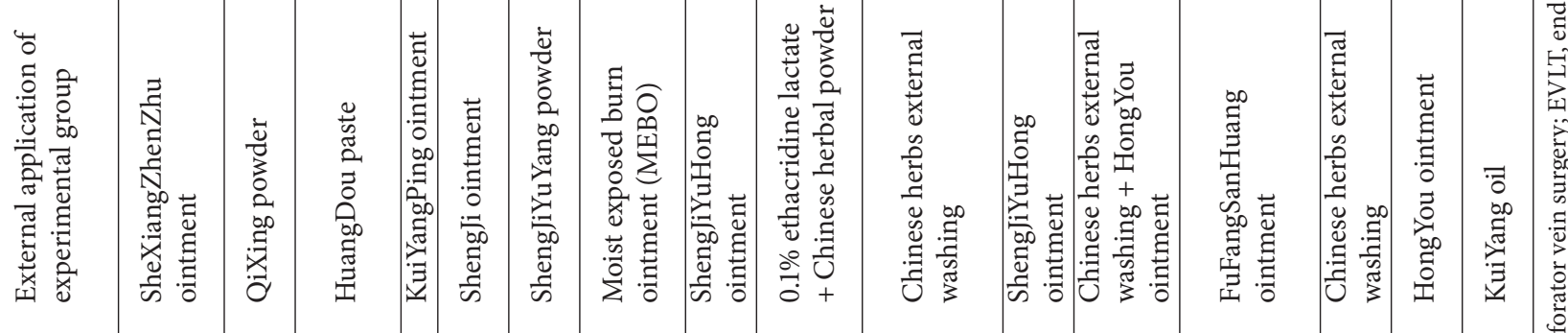

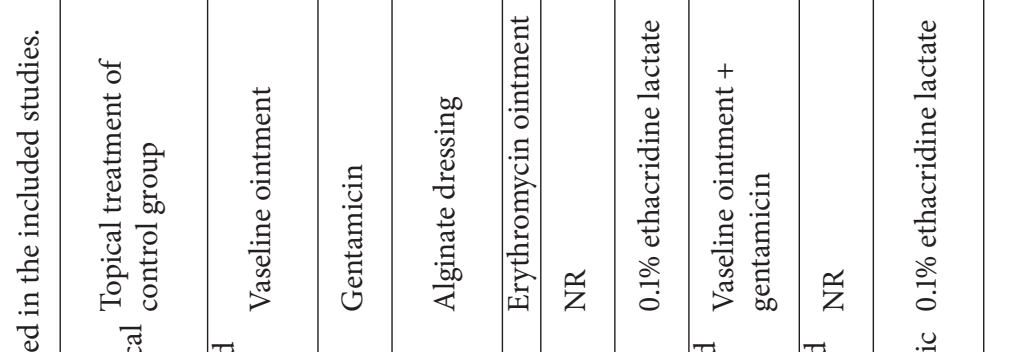

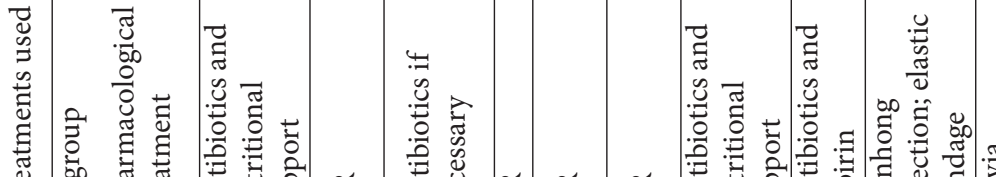

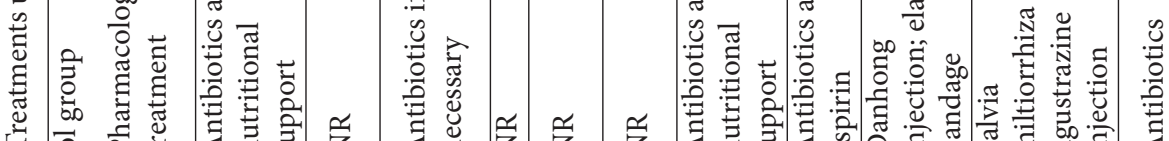

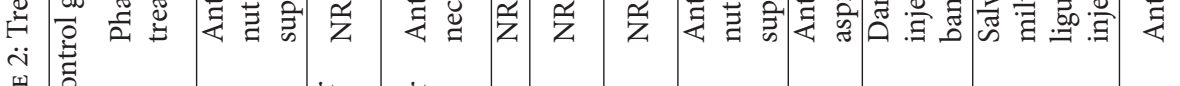

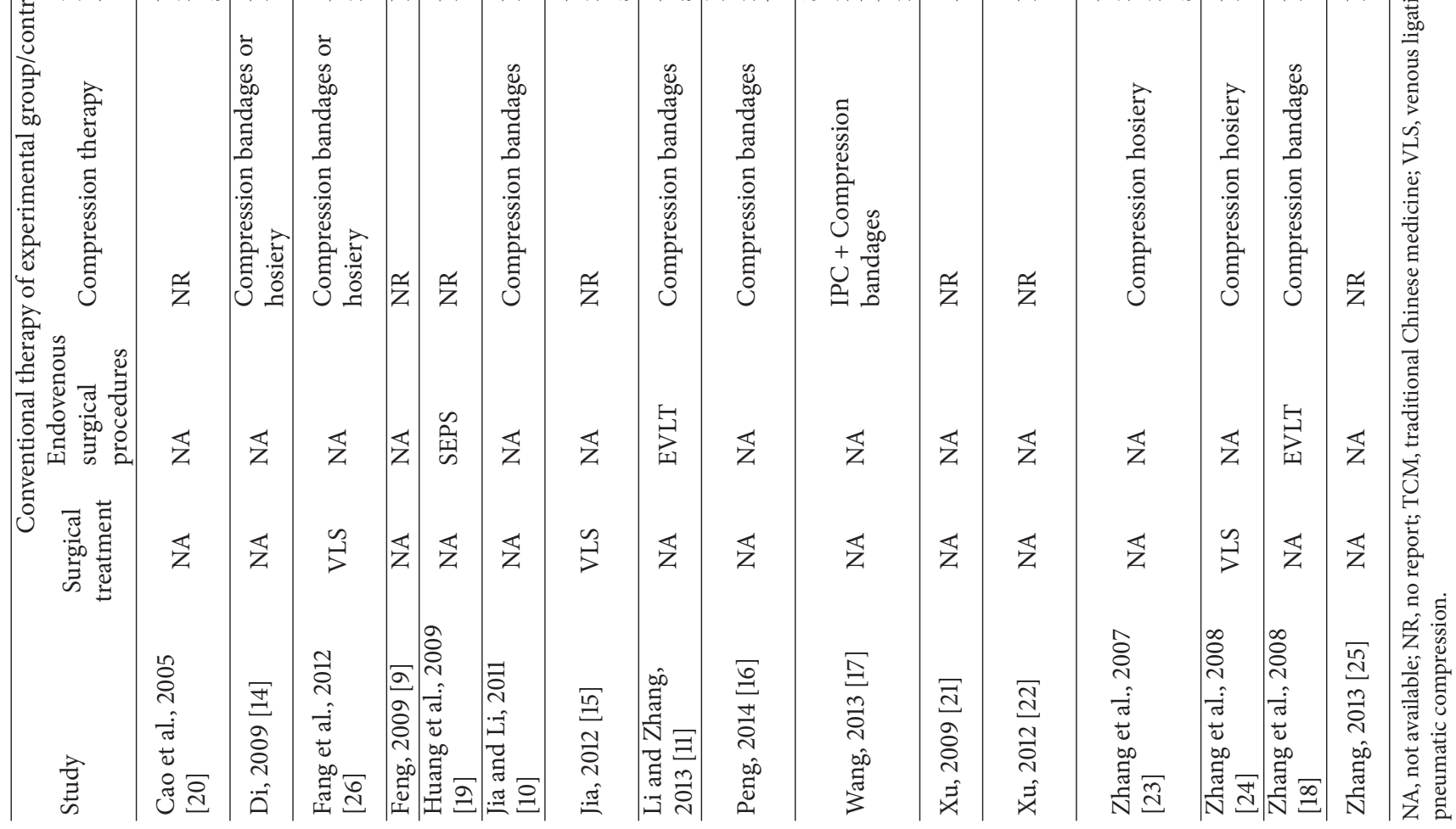




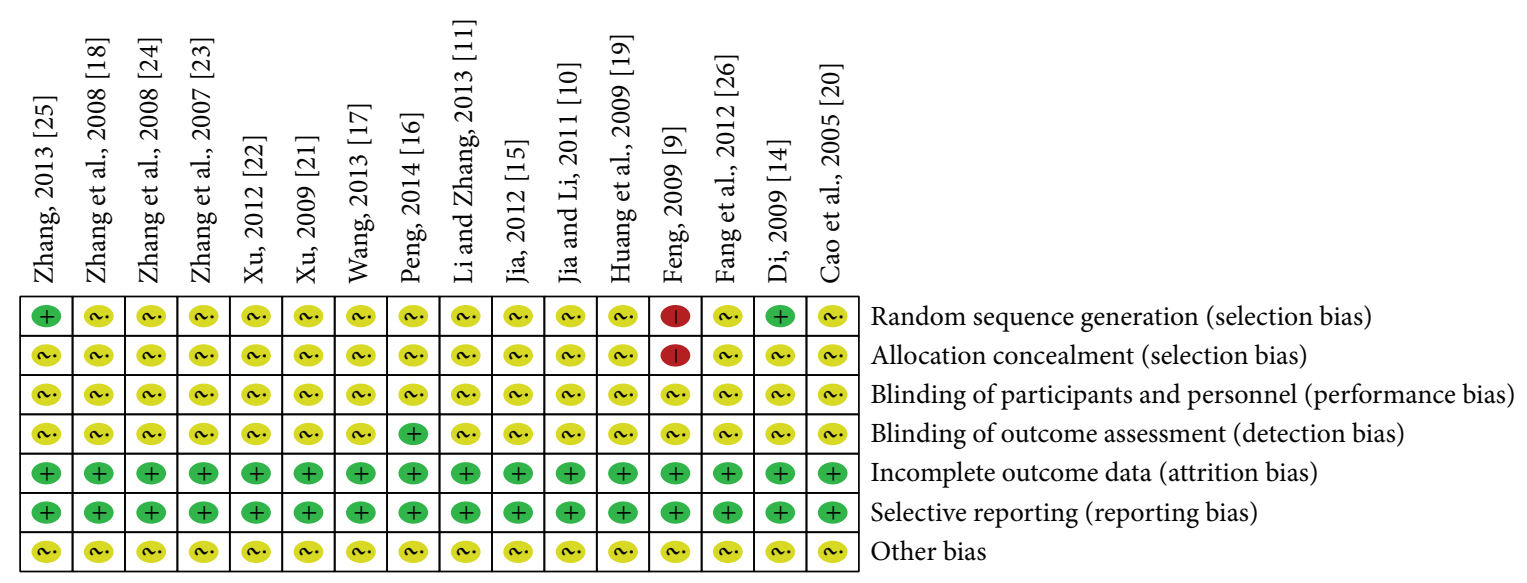

FIgURE 2: Risk of bias graph.

There were also significant differences in each subgroup (basic intervention strategies: first-line clinical treatment RR $=1.27,95 \% \mathrm{CI}=1.18-1.37$; basic intervention strategies: firstline and surgical treatment $\mathrm{RR}=1.13,95 \% \mathrm{CI}=1.05-1.22$ ) (Figure 3).

\subsubsection{Total Effectiveness Rates of EA-TCM Combined with} Conventional Therapy versus Conventional Therapy Alone. Five studies with 314 subjects reported that the experimental groups received EA-TCM combined with conventional therapy and that the control groups received conventional therapy only. Results of meta-analysis using the fixed-effects model indicated a significantly higher total effectiveness rate for EATCM combined with conventional therapy compared to that of the control groups $(\mathrm{RR}=1.11,95 \% \mathrm{CI}=1.04-1.19$, and $P=$ $0.003)$. Significant differences were found between subgroups of conventional therapy with first-line clinical treatment $(\mathrm{RR}=1.14,95 \% \mathrm{CI}=1.00-1.31)$ and conventional therapy with first-line clinical treatment combined with surgical interventions $(\mathrm{RR}=1.09,95 \% \mathrm{CI}=1.01-1.17)$ (Figure 4).

3.4.3. Recurrence Rate Sat Followup. Three studies reported recurrence rates at followup. However, the results of metaanalysis using the fixed-effects model indicated no significant effects in the experimental groups compared to the control groups $(\mathrm{RR}=0.86,95 \% \mathrm{CI}=0.31-2.39$, and $P=0.85)$ (Figure 5).

3.5. Secondary Outcomes. Only two studies reported final pain measurements. With random-effects modelling, the pooled data for the two studies showed a difference between the experimental and control groups in the final pain measurements $(\mathrm{MD}-0.75,95 \% \mathrm{CI}=-2.15-0.65$, and $P=0.29)$ (Figure 6).

3.6. Adverse Events. Six studies reported adverse events in the experimental groups. No significant adverse reactions were noted in three studies [15-17]. Two trials reported two patients with contact dermatitis that resolved with appropriate treatment $[14,18]$. Three patients suffered from ecchymosis caused by surgical treatment [11].

3.7. Assessment of Publication Bias. In this review, the use of funnel plots was limited due to the small number of studies evaluated.

\section{Discussion}

4.1. Summary of Evidence. This review systematically assessed mainly Chinese-sourced RCT studies related to the effects of EA-TCM as a complementary therapy; a total of 16 RCTs were identified for systematic review and meta-analysis. The trials included in this study assessed the efficacy of several types of external application on various medical conditions. A total of 660 patients in treatment groups and 609 in control groups were evaluated, and the duration of RCTs ranged from 6.4 months to 10.5 years. All of these RCTs were conducted in mainland China. Despite the fact that most of the trials had small sample sizes and poor methodological quality, analysis of the pooled data showed a consistently superior effect of EA-TCM or EA-TCM combined with conventional therapy in terms of total effectiveness, when compared to the control groups. There were fewer adverse effects, and none were severe; only two trials mentioned any adverse effects of EA-TCM, in which two patients each presented with slight rashes [14, 18]. No patients dropped out of their trials due to adverse effects, suggesting that EA-TCM is safe for clinical use.

4.2. Limitations of This Review. As with all such studies, we acknowledge several limitations. Specifically, the distorting effects of publication and location bias on systematic reviews and meta-analyses have been well documented [27]. Although we are confident that our search strategy located all relevant studies, there remains a certain degree of uncertainty. The quality scores of the included RCTs were generally poor. Although all of the included studies had a randomization design, only three described the details of the randomization 


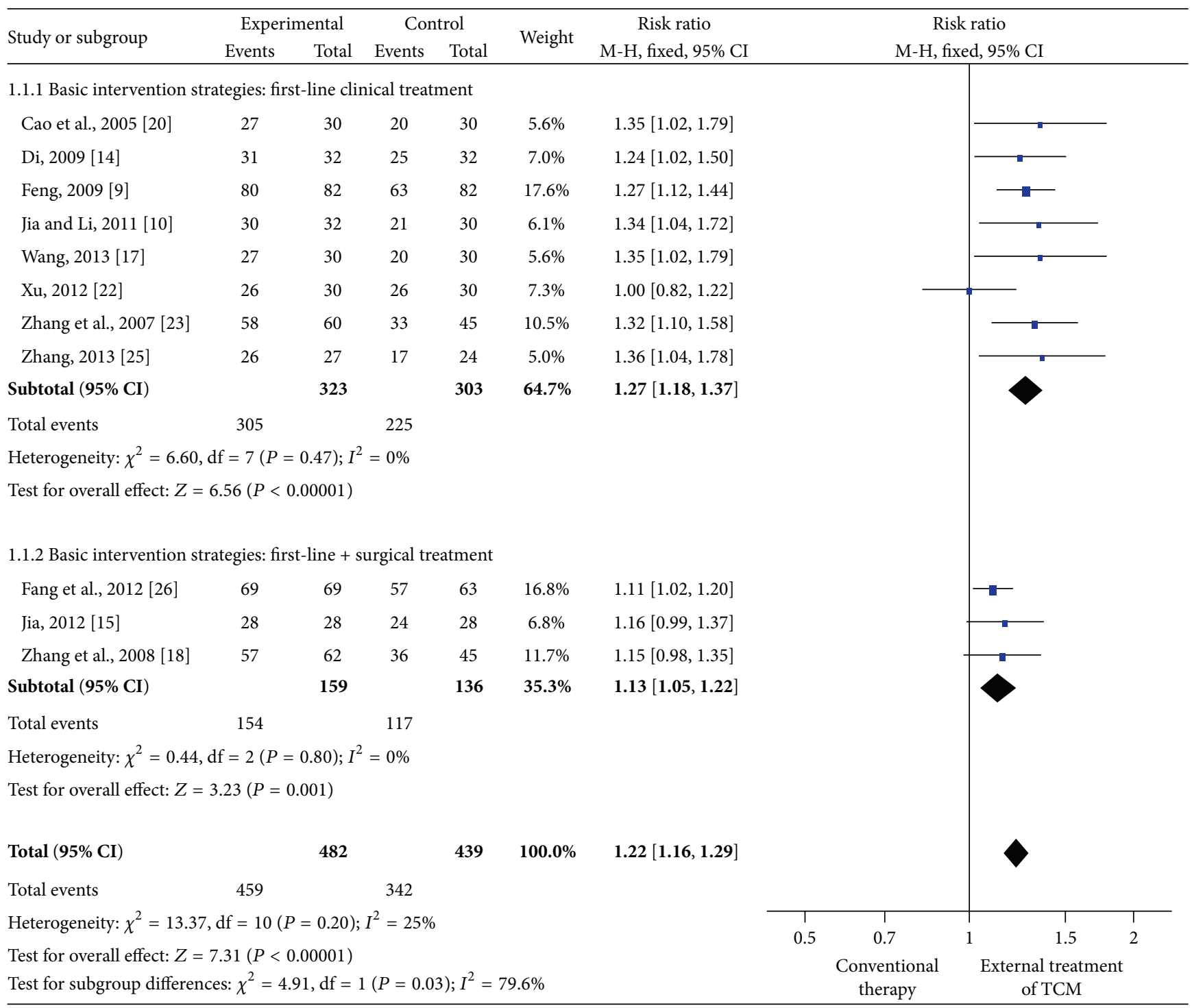

FIGURE 3: Meta-analysis of the total effectiveness rate of external application of traditional Chinese medicine (EA-TCM) versus conventional therapy based on the same intervention strategies. CI indicates confidence interval.

$[9,14,25]$. Furthermore, information on allocation concealment or participant and personnel blinding was missing, and only one study reported any details of the blinding of outcome assessments [16].

While Cochrane's $\chi^{2}$ and $I^{2}$ tests revealed no statistical heterogeneity in the total effectiveness rate among these studies, an unpredictable clinical heterogeneity was present nonetheless. For example, basic intervention strategies and conventional therapies, components of TCM, wound-cleaning methods, and care approaches were different in each RCT.

4.3. Possible Rationales for EA-TCM for Treatment of Venous Ulcers. As a type of chronic skin ulcer, the pathogenesis of venous ulcers is theoretically caused by "Re (heat) evil," "Yu (qi-stagnancy, blood-stasis)," and "Xu (qi blood and yin yang deficiency)" according to stage. Correspondingly, the treatment principles for the three stages of venous ulcer include clearing away heat and dampness, promoting blood circulation to dissipate blood stasis, and providing supplements for deficiencies [28]. Although the components of EATCM used in each trial included in our meta-analysis varied, the treatment principles were consistent: SheXiangZhenZhu ointment [20], QiXing powder [14], ShengJi ointment [19], moist exposed burn ointment [15], and Chinese herbs for external washing [17] all clear away heat and dampness and promote blood circulation to dissipate blood stasis. For example, the main effect of HuangDou paste [26] is to clear away heat and address deficiencies; other treatments $[9,11,16$, 18, 21-23] also offer combined effects for the three treatment stages.

As an important complementary therapy, the use of EATCM combined with conventional therapy could offer an 


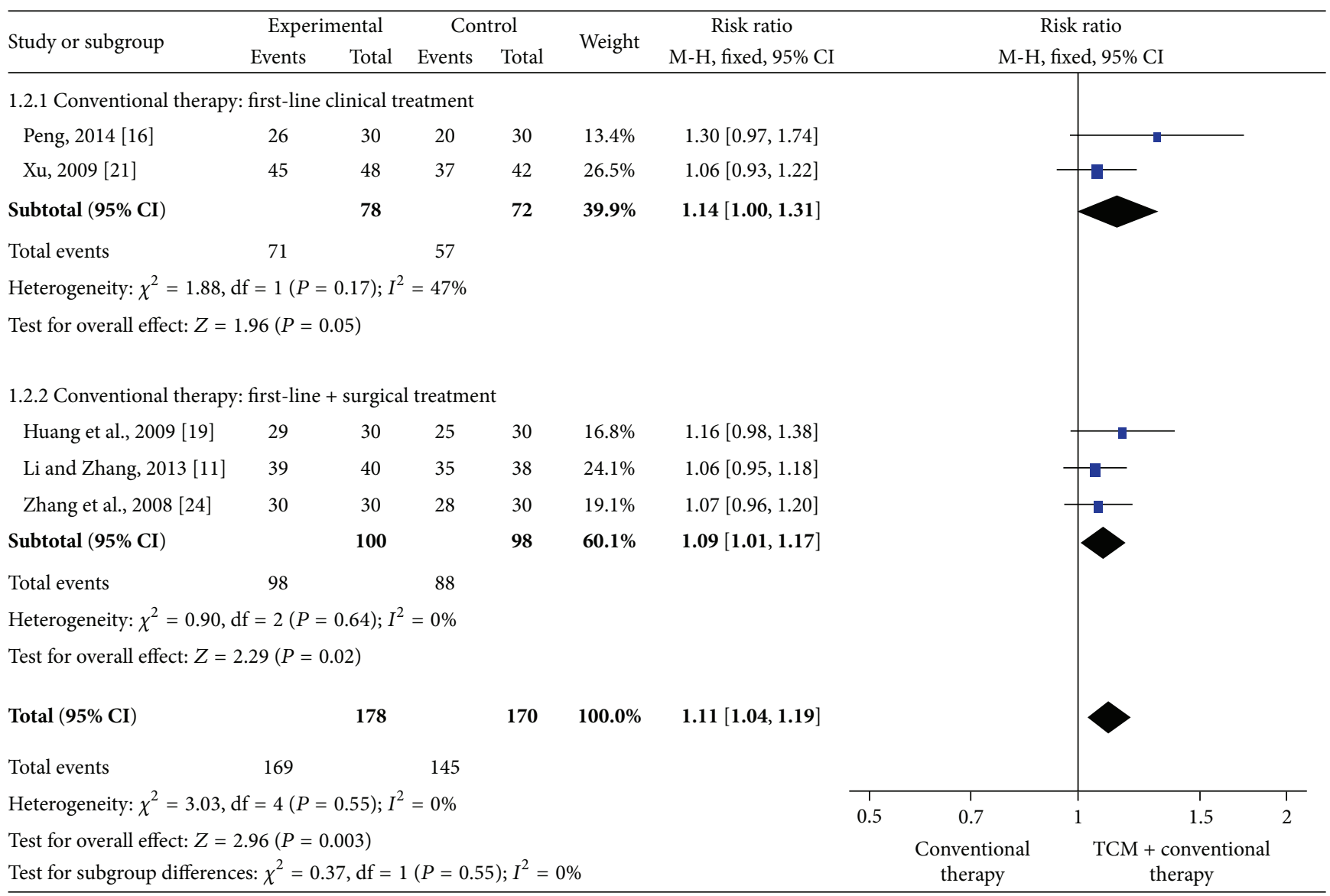

FIgURE 4: Meta-analysis of the total effectiveness rate of combined external application of traditional Chinese medicine (EA-TCM) and conventional therapy versus conventional therapy alone. CI indicates confidence interval.

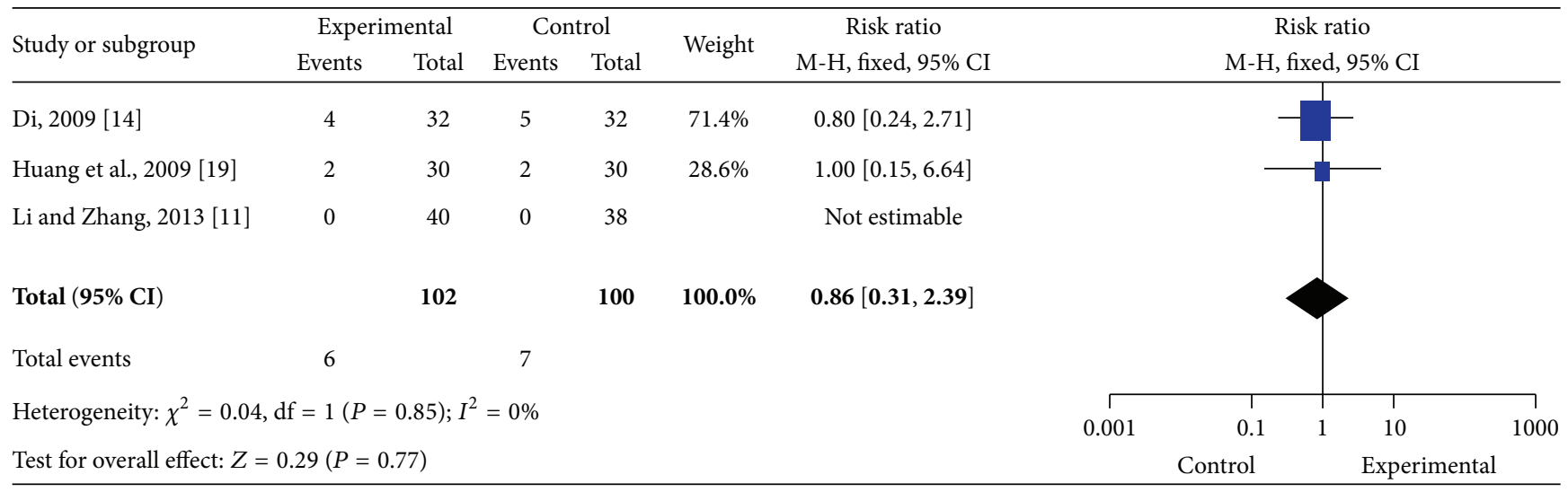

FIGURE 5: Meta-analysis of recurrence rates during followup. CI indicates confidence interval.

effective treatment method for venous ulcers. Therefore, a substantial amount of research has investigated the chemical constituents of EA-TCMs. Moist exposed burn ointment, the most representative EA-TCM, has pharmacological effects that include prevention of dermal water loss, as well as antiinflammatory, antibacterial, and analgesic properties [2931]. ShengJiYuHong ointment has been shown to inhibit inflammatory responses by acting on the inflammatory mediators and cells and to improve wound healing by promoting fibroblast proliferation and tissue granulation [32-34]. Wang et al. found that HongYou ointment offers a protective effect on the production and secretion of the extracellular matrix and also promotes fibroblast and endothelial cell proliferation [35]. 


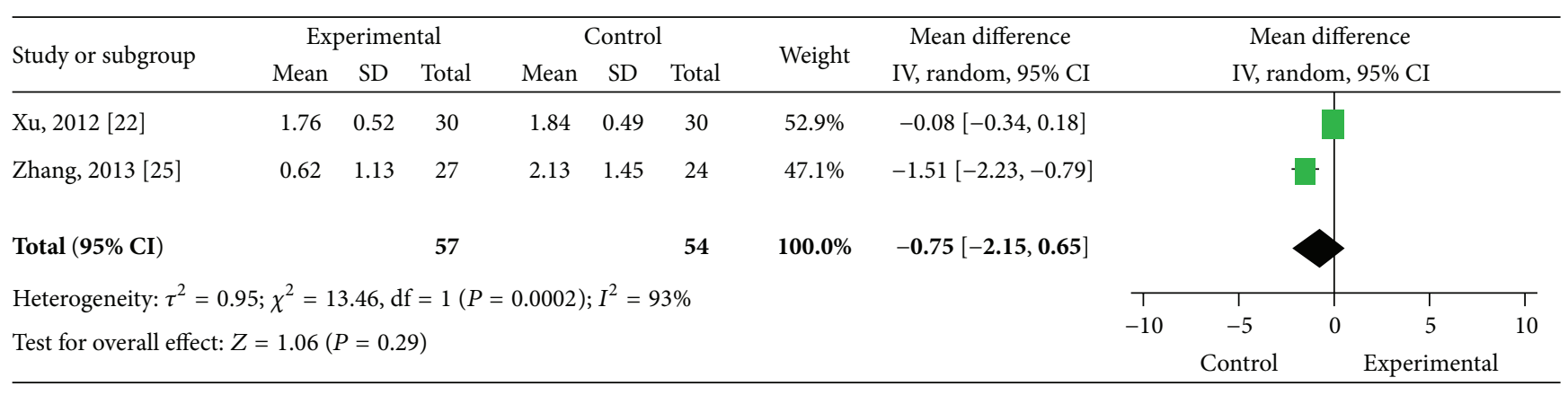

FIGURE 6: Meta-analysis of final pain measurements. CI indicates confidence interval.

\section{Conclusions}

While the evidence that EA-TCM may be an effective treatment for venous ulcers is encouraging, it is not conclusive due to the low methodological quality of the RCTs. Therefore, more high-quality RCTs, with low risk of bias and adequate sample sizes, are required to demonstrate its true effects.

\section{Conflict of Interests}

The authors declare that there is no conflict of interests regarding the publication of this paper.

\section{Authors' Contributions}

Xin Li, Qing-qing Xiao, Kan Ze, Su Li, Yi-fei Wang, Min Zhou, Fu-lun Li, and Bin Li had full access to all study data and take responsibility for its integrity and the accuracy of the analysis. Xin Li, Fu-lun Li, and Bin Li were responsible for the study concept and design. Xin Li and Qing-qing Xiao were responsible for data acquisition, and Kan $\mathrm{Ze}$ and $\mathrm{Su} \mathrm{Li}$ were responsible for data extraction. The assessment of bias risk was performed by Xin Li and Min Zhou; data analysis and interpretation were performed by Xin Li, Yi-fei Wang, and $\mathrm{Fu}$-lun Li. The paper was drafted by Xin Li, Fu-lun Li, and Bin Li, while Xin Li and Qing-qing Xiao provided critical paper review for important intellectual content. Statistical analyses were performed by Xin Li and Kan Ze, and Fu-lun Li and Bin Li supervised the study.

\section{Acknowledgments}

This study was supported by a grant from the National Science Foundation (NSFC) of China (no. 81373648 to Fulun Li, 81273764 and 81473682 to Bin Li, and 81302971 to Xin $\mathrm{Li}$ ). It was also supported by grants from the Shanghai Science and Technology Committee (no. 12401903500 to Bin Li, 12QA1403300 to Fu-lun Li, and 14401972703 to Xin Li); the Shanghai Health Bureau project (ZYSNXD-CC-ZDYJ018, ZY3-CCCX-1-1008, XYQ2013073 and 2012QL005A); training program of Shanghai youth university teachers to Kan Ze.

\section{References}

[1] D. J. Margolis, J. A. Berlin, and B. L. Strom, "Which venous leg ulcers will heal with limb compression bandages?" The American Journal of Medicine, vol. 109, no. 1, pp. 15-19, 2000.

[2] R. Word, "Medical and surgical therapy for advanced chronic venous insufficiency," Surgical Clinics of North America, vol. 90, no. 6, pp. 1195-1214, 2010.

[3] J. J. Bergan, G. W. Schmid-Schönbein, P. D. Coleridge Smith, A. N. Nicolaides, M. R. Boisseau, and B. Eklof, "Chronic venous disease," The New England Journal of Medicine, vol. 355, no. 5, pp. 488-498, 2006.

[4] L. P. Fernandes Abbade and S. Lastória, "Venous ulcer: epidemiology, physiopathology, diagnosis and treatment," International Journal of Dermatology, vol. 44, no. 6, pp. 449-456, 2005.

[5] I. Flessenkämper, M. Hartmann, K. Hartmann, D. Stenger, and S. Roll, "Endovenous laser ablation with and without high ligation compared to high ligation and stripping for treatment of great saphenous varicose veins: results of a multicentre randomised controlled trial with up to 6 years follow-up," Phlebology, 2014.

[6] P. Zamboni, C. Cisno, F. Marchetti et al., "Minimally invasive surgical management of primary venous ulcers vs. compression treatment: a randomized clinical trial," European Journal of Vascular and Endovascular Surgery, vol. 25, no. 4, pp. 313-318, 2003.

[7] J. R. Barwell, C. E. Davies, J. Deacon et al., "Comparison of surgery and compression with compression alone in chronic venous ulceration (ESCHAR study): randomised controlled trial," The Lancet, vol. 363, no. 9424, pp. 1854-1859, 2004.

[8] S. Subramonia and T. Lees, "Radiofrequency ablation vs conventional surgery for varicose veins-a comparison of treatment costs in a randomised trial," European Journal of Vascular and Endovascular Surgery, vol. 39, no. 1, pp. 104-111, 2010.

[9] D. X. Feng, Clinical efficacy of the KuiYangping ointment for venous leg ulcers [M.S. thesis], Liaoning University of Traditional Chinese Medicine, 2009.

[10] L. H. Jia and Y. Q. Li, "Clinical observation of ShengJiYuYang powder treatment for venous leg ulcers," in Proceedings of the 2nd Conference of Peripheral Vascular Disease, Academic Association of Chinese Medicine, 2011.

[11] K. S. Li and Z. Y. Zhang, "Clinical observation of endovenous laser treatment combined with Chinese herbs external application for venous leg ulcers of 40 patients," Guiding Journal of 
Traditional Chinese Medicine and Pharmacy, vol. 19, no. 2, pp. 49-50, 2013.

[12] J. Ezzo, B. M. Berman, A. J. Vickers, and K. Linde, "Complementary medicine and the cochrane collaboration," The Journal of the American Medical Association, vol. 280, no. 18, pp. 16281630, 1998.

[13] J. P. T. Higgins, D. G. Altman, P. C. Gøtzsche et al., "The cochrane collaboration's tool for assessing risk of bias in randomised trials," The British Medical Journal, vol. 343, Article ID d5928, 2011.

[14] D. K. Di, Clinical observation to QiXing Dan on the treatment of venous leg ulcers [M.S. thesis], Chengdu University of Traditional Chinese Medicine, Chengdu, China, 2009.

[15] K. X. Jia, "Clinical study of moist exposed burn ointment on the treatment of venous leg ulcers," Medical Frontier, vol. 2, no. 30, pp. 206-207, 2012.

[16] B. Peng, Chinese medicine internal and external treatment of venous leg ulcer treatment efficacy of clinical studies [M.S. thesis], Hebei Medical University, Shijiazhuang, China, 2014.

[17] W. W. Wang, The clinical research of the effect of treatment of venous ulcers treated by Chinese Herbs wash and conventional therapy [M.S. thesis], Hebei Medical University, Shijiazhuang, China, 2013.

[18] L. Zhang, G. B. Liu, S. Q. Yan, and X. Yang, "Treatment of 62 cases of ulcers cruris by HongYou Plaster and endovenous laser treatment at superficial veins of lower limbs," Shanghai Journal of Traditional Chinese Medicine, vol. 42, no. 7, pp. 57-58, 2008.

[19] K. J. Huang, L. W. Yang, and D. R. Li, "Clinical study of subfascial endoscopic perforator vein surgery combined with ShengJi ointment for venous leg ulcers," Information on Traditional Chinese Medicine, vol. 26, no. 2, pp. 62-63, 2009.

[20] R. W. Cao, H. J. An, and D. P. Cao, "Clinical observation of the external treatment of SheXiangZhenZhu ointment for venous leg ulcers of 60 patients," Chinese General Practice, vol. 8, no. 18, p. $1547,2005$.

[21] Z. Q. Xu, "Clinical efficacy of the external treatment of ShengjiYuHong ointment for venous leg ulcers of 90 patients," in Proceedings of the 4th Symposium of Peri Operation Period of National Integrative Medicine, 2009.

[22] W. J. Xu, "Nursing observation of HongYou ointment combined with Chinese herbs external treatment for venous leg ulcers," China Health Care Nutrition, vol. 23, no. 11, pp. 169-170, 2012.

[23] J. Q. Zhang, H. J. Li, S. W. Wang, and J. Li, "The treatment for venous leg ulcers of 60 patients by FuFangSanHuang ointment," Chinese Journal of Surgery of Integrated Traditional and Western Medicine, vol. 13, no. 6, pp. 537-539, 2007.

[24] H. Zhang, J. Yang, W. Li, and H. Y. Hu, "Clinical efficacy of the external treatment of Chinese herbs for venous leg ulcers of 30 patients," Yunnan Journal of Traditional Chinese Medicine and Materia Medica, vol. 29, no. 5, pp. 15-16, 2008.

[25] W. W. Zhang, Clinical efficacy of the external treatment of KuiYang oil for venous leg ulcers [M.S. thesis], Beijing University of Chinese Medicine, 2013.

[26] X. H. Fang, H. P. Li, X. Fang, and Y. Jin, “Observation on curative effect of external application with soybean paste for patients with chronic venous in lower limb," Chinese Nursing Research, vol. 26, no. 8, pp. 2109-2111, 2012.

[27] M. Egger and G. D. Smith, "Bias in location and selection of studies," British Medical Journal, vol. 316, no. 7124, pp. 61-66, 1998.
[28] F. L. Li, Y. F. Wang, X. Li et al., "Characteristics and clinical managements of chronic skin ulcers based on traditional Chinese medicine," Evidence-Based Complementary and Alternative Medicine, vol. 2012, Article ID 930192, 6 pages, 2012.

[29] B. S. Atiyeh, R. Dham, M. Kadry et al., "Benefit-cost analysis of moist exposed burn ointment," Burns, vol. 28, no. 7, pp. 659-663, 2002.

[30] B. S. Atiyeh, J. Ioannovich, G. Magliacani et al., "Efficacy of moist exposed burn ointment in the management of cutaneous wounds and ulcers: a multicenter pilot study," Annals of Plastic Surgery, vol. 48, no. 2, pp. 226-227, 2002.

[31] M. R. EL-Hadidy, A. R. EL-Hadidy, A. Bhaa, S. A. Asker, and S. A. Mazroa, "Role of epidermal stem cells in repair of partialthickness burn injury after using Moist Exposed Burn Ointment (MEBO) histological and immunohistochemical study," Tissue and Cell, vol. 46, no. 2, pp. 144-151, 2014.

[32] Y. Chen, ShengJiYuHong ointment cured rat myogenic and promote wound healing clinical and experimental research [M.S. thesis], Nanjing University of Chinese Medicine, 2012.

[33] X. P. Dong, L. J. Wang, H. Yi et al., "Experimental study of Shengji Yuhong Ointment on analgesic and anti-inflammatory actions," Journal of Gansu College of Traditional Chinese Medicine, vol. 28, no. 1, pp. 10-12, 2011.

[34] Y. Zhou, The clinical and animal research of ShengJiYuHong ointment on wound healing by promoting the growth of granulation [M.S. thesis], Nanjing University of Chinese Medicine, 2012.

[35] Y. Wang, M. Zhang, H. Y. Shi, and M. Xiao, "Empirical study on influence of Hongyougao on wound healing in rats," Modern Journal of Integrated Traditional Chinese and Western Medicine, vol. 19, no. 11, pp. 1313-1314, 2010. 


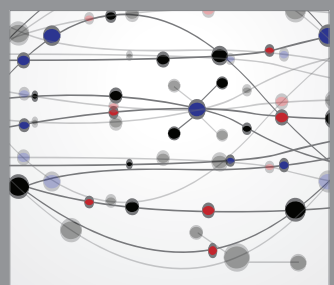

The Scientific World Journal
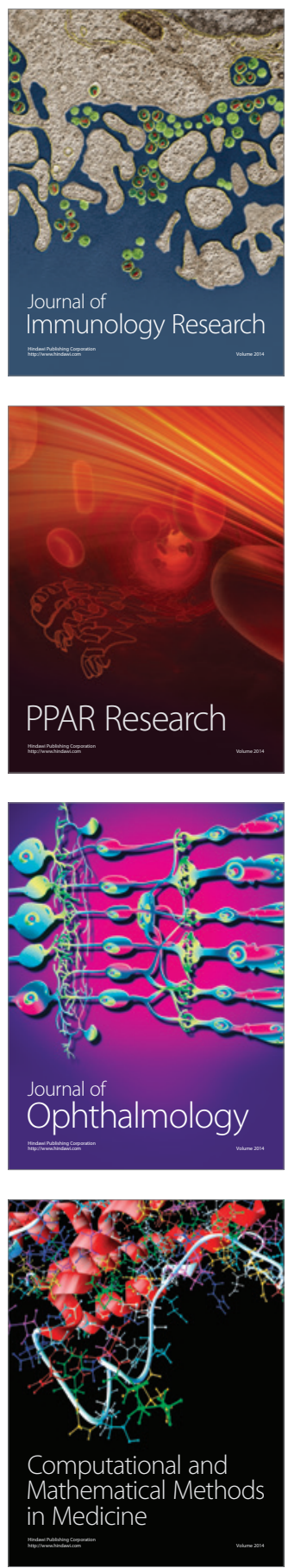

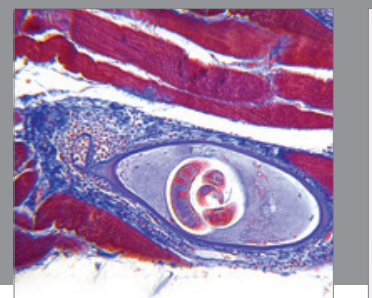

Gastroenterology

Research and Practice
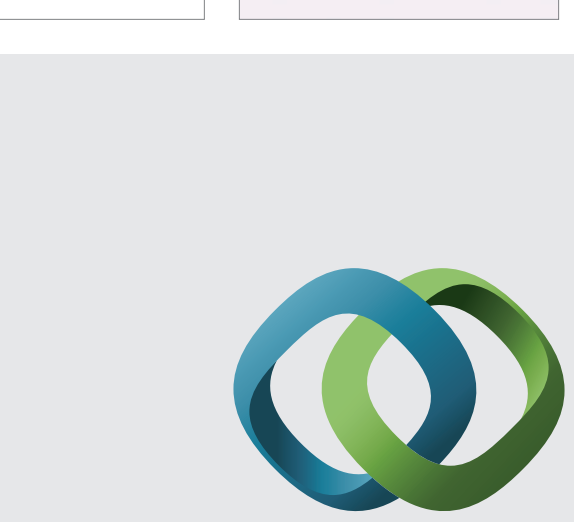

\section{Hindawi}

Submit your manuscripts at

http://www.hindawi.com
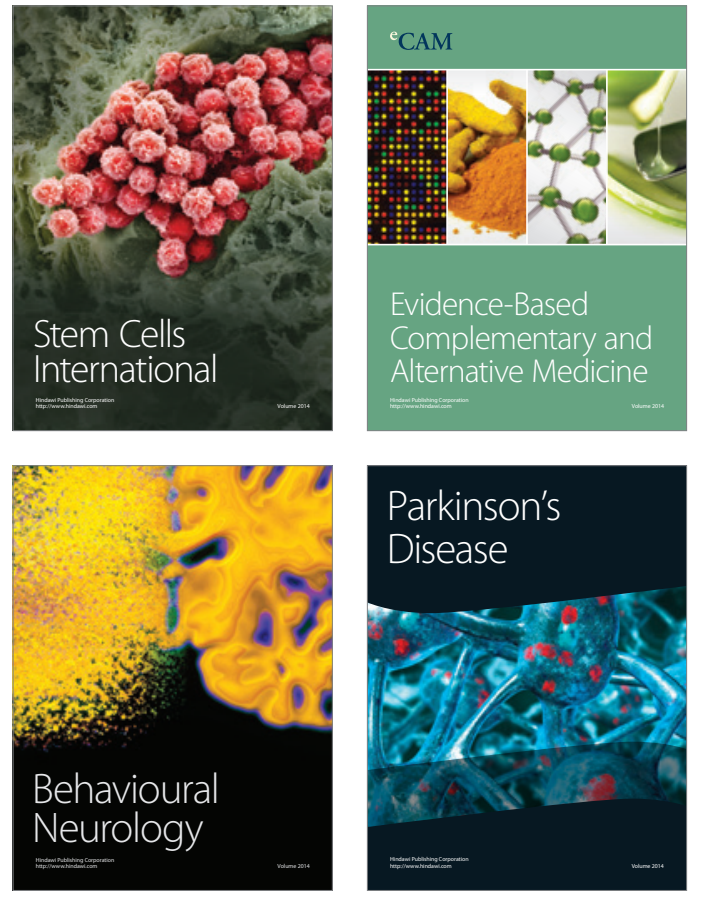
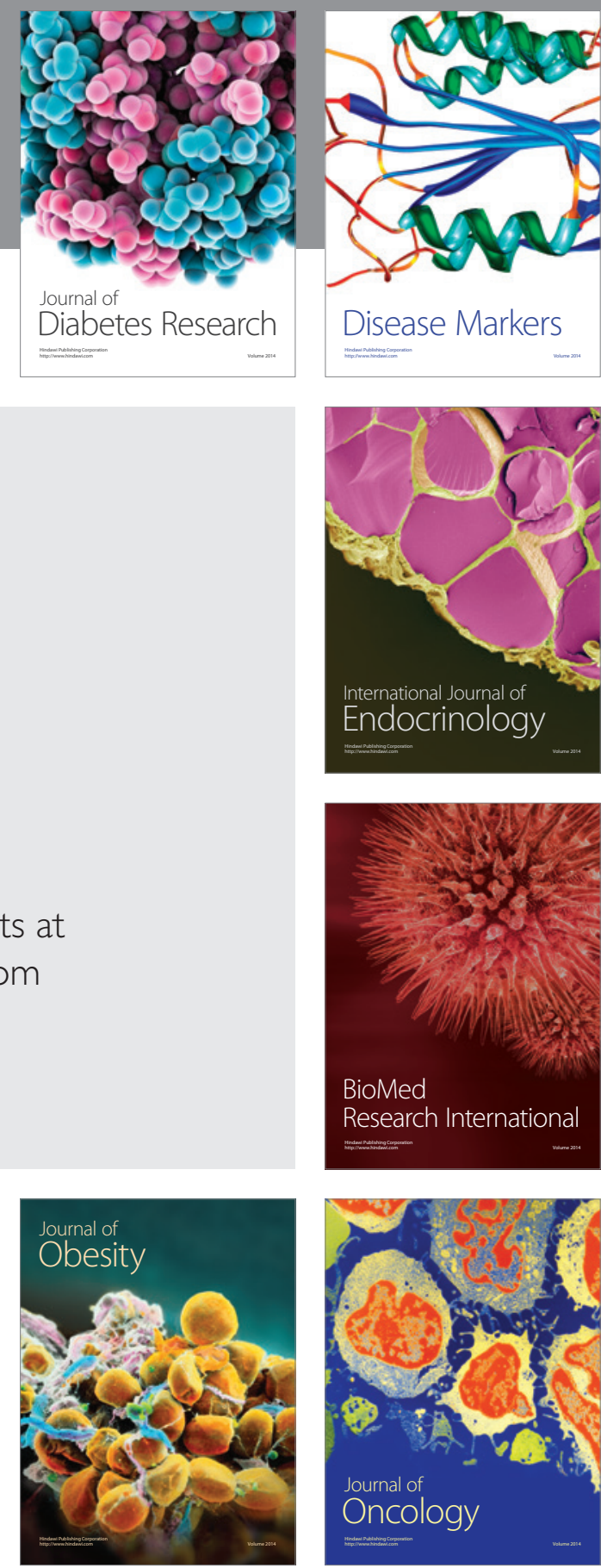

Disease Markers
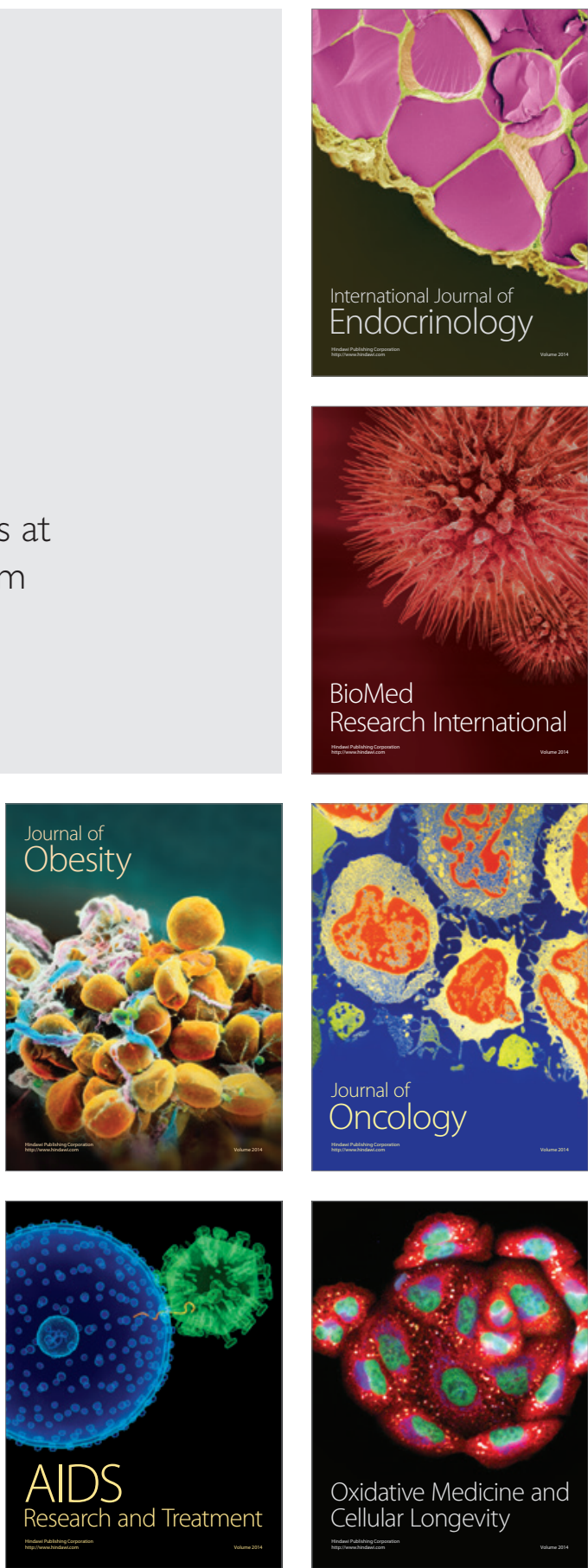